\title{
Introduction to the special issue on artificial intelligence in musculoskeletal radiology
}

\author{
Connie Y. Chang ${ }^{1} \cdot$ Thomas M. Link ${ }^{2}$
}

Published online: 8 October 2021

(c) ISS 2021

Artificial intelligence (AI) and Radiology are at an interesting inflection point. Prior research has been focused on developing the technology, and slowly but surely ideas are becoming practical applications, with more and more scientific papers focused on tackling clinical problems and integration into our day-to-day workflow becoming a closer reality. In this special issue, our goal was to bring you upto-date information about the progress that has been made in musculoskeletal AI applications and also to help you to get a "picture" of what is coming soon. At the end of each review article, there is a "picture this" section-which sets the stage for how AI could become a part of our daily clinical work. We hope that these glimpses into our potential future will excite you as much as they have excited us. We also hope that the clinical-based approach in these articles will equip you to understand the AI technologies as they are released and implemented in the coming years.

Over the course of the past year, this special issue has morphed into an exciting collection of perspectives, review articles, and scientific papers. We cover the past, present, and future and even touch upon relevant legal issues. We have authors from 6 countries and 19 institutions. We have seasoned researchers and clinicians as well as fresh talent. Manuscripts cover a range of topics, including trauma, orthopedic implants, spine imaging, oncology, and osteoarthritis. They also provide insights into the potential impact of AI on daily operations and MRI imaging and interpretation. We will provide a primer on how to get started with AI clinical applications and finally experts in the field will review current and future perspectives. We are very grateful to our authors and contributors for providing a

Connie Y. Chang

cychang@mgh.harvard.edu

1 Massachusetts General Hospital and Harvard Medical School, 55 Fruit Street Yawkey 6E, Boston, MA 02114, USA

2 University of California at San Francisco, San Francisco, CA, USA state-of-the-art and multifaceted coverage of this exciting topic. AI scientific submissions to Skeletal Radiology have been steadily increasing, and we believe that our journal is ideally suited to engage in conversation about AI clinical applications specific to Musculoskeletal Radiology and Intervention. We hope that this special issue will initiate an ongoing conversation, and we encourage Musculoskeletal Radiologists engaged in AI research to continue to submit their high-quality work to our journal.

Finally, we are honored to be part of this inaugural effort to integrate Special Issues and Special Sections in Skeletal Radiology. I (Connie) especially want to take this opportunity to thank the Editors for their mentorship and guidance throughout this exciting year as the inaugural Skeletal Radiology Editorial Fellow. This one-year fellowship was a wonderful learning experience and has provided me with important insights into the role of Skeletal Radiology in the MSK scientific community. It was also a unique career forming experience and has heightened my excitement to pursue an academic career. Thank you very, very much!

\section{Declarations}

Ethical approval This article does not contain any studies with human participants performed by the authors.

Conflict of interest The authors declare that they have no conflict of interest.

Publisher's Note Springer Nature remains neutral with regard to jurisdictional claims in published maps and institutional affiliations. 